\title{
Teaching NeuroImages: Acute convexity subarachnoid hemorrhage
}

\author{
An underrecognized presentation of CAA-ri
}

Aikaterini Theodorou, MD, Stefanos Lachanis, MD, Palaiologos Alexopoulos, MD, Lina Palaiodimou, MD, Nikoletta Kollia, MD, Konstantinos Voumvourakis, MD, PhD, and Georgios Tsivgoulis, MD, PhD, MSc, FESO

Neurology ${ }^{\circledR}$ 2019;93:e524-e525. doi:10.1212/WNL.0000000000007873

Nontraumatic acute convexity subarachnoid hemorrhage (cSAH) is a common manifestation of cerebral amyloid angiopathy (CAA), but has been rarely reported in patients with CAArelated inflammation (CAA-ri). ${ }^{1,2}$

We describe the neuroimaging findings of an 89-year-old woman presenting with a 1-month history of visual hallucinations, disorientation, and agitation. Brain MRI (figure, A-F) showed disseminated cortical superficial siderosis, cerebral microbleeds, and asymmetric white matter hyperintensities in both hemispheres. There was also subacute cSAH in right parietal lobe with diffusion restriction and gadolinium enhancement in subarachnoid space.

The patient fulfilled CAA-ri diagnostic criteria and was treated with IV methylprednisolone with clinical and radiologic (figure, G) improvement.

\section{Study funding}

No targeted funding reported.

Figure Imaging
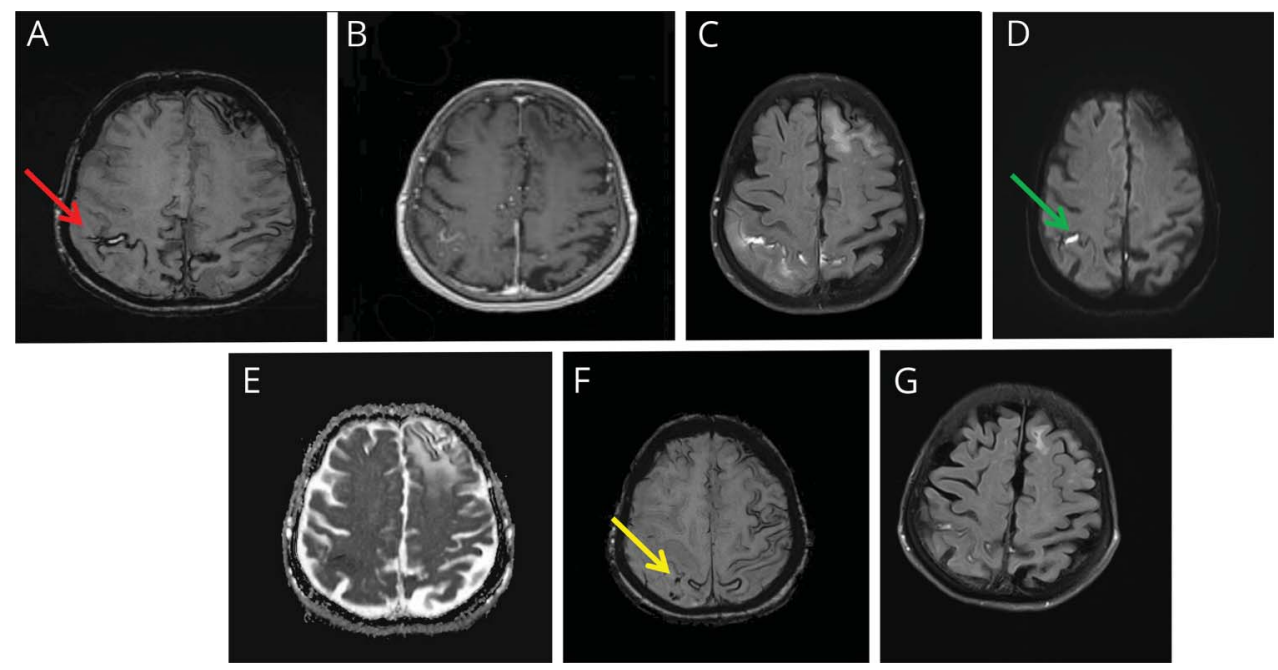

Axial susceptibility-weighted imaging (SWI) shows convexity subarachnoid hemorrhage (red arrow, A). There was evidence of gadolinium enhancement in the subarachnoid space (B) and asymmetric white matter hyperintensities on fluid-attenuated inversion recovery sequences (C). Diffusion restriction was documented on right parietal lobe (green arrow, D; E). Cortically located cerebral microbleeds were also documented on SWI (yellow arrow, F). Right parietal white matter hyperintensities resolved following corticosteroid treatment (G).

\section{Correspondence}

Dr. Theodorou

katetheo24@gmail.com

\section{MORE ONLINE}

\section{$\rightarrow$ Teaching slides}

links.lww.com/WNL/

A935

From the Second Department of Neurology (A.T., P.A., L.P., N.K., K.V., G.T.), National and Kapodistrian University of Athens, School of Medicine, "Attikon" University Hospital; latropolis Magnetic Resonance Diagnostic Centre (S.L.), Athens, Greece; and Department of Neurology (G.T.), The University of Tennessee Health Science Center, Memphis.

Go to Neurology.org/N for full disclosures. Funding information and disclosures deemed relevant by the authors, if any, are provided at the end of the article. 


\section{Disclosure}

The authors report no disclosures relevant to the manuscript. Go to Neurology.org/N for full disclosures.

Appendix Authors

\begin{tabular}{|c|c|c|c|}
\hline Name & Location & Role & Contribution \\
\hline $\begin{array}{l}\text { Aikaterini } \\
\text { Theodorou, MD }\end{array}$ & $\begin{array}{l}\text { "Attikon" University } \\
\text { Hospital, Athens, } \\
\text { Greece }\end{array}$ & Author & $\begin{array}{l}\text { Drafting and } \\
\text { revising the } \\
\text { manuscript }\end{array}$ \\
\hline $\begin{array}{l}\text { Stefanos } \\
\text { Lachanis, MD }\end{array}$ & $\begin{array}{l}\text { latropolis Magnetic } \\
\text { Resonance } \\
\text { Diagnostic Centre, } \\
\text { Athens, Greece }\end{array}$ & Author & $\begin{array}{l}\text { Data collection, } \\
\text { critical comments } \\
\text { during } \\
\text { manuscript } \\
\text { revision }\end{array}$ \\
\hline $\begin{array}{l}\text { Palaiologos } \\
\text { Alexopoulos, } \\
\text { MD }\end{array}$ & $\begin{array}{l}\text { "Attikon" University } \\
\text { Hospital, Athens, } \\
\text { Greece }\end{array}$ & Author & $\begin{array}{l}\text { Critical comments } \\
\text { during } \\
\text { manuscript } \\
\text { revision }\end{array}$ \\
\hline $\begin{array}{l}\text { Lina } \\
\text { Palaiodimou, } \\
\text { MD }\end{array}$ & $\begin{array}{l}\text { "Attikon" University } \\
\text { Hospital, Athens, } \\
\text { Greece }\end{array}$ & Author & $\begin{array}{l}\text { Data collection, } \\
\text { critical comments } \\
\text { during } \\
\text { manuscript } \\
\text { revision }\end{array}$ \\
\hline
\end{tabular}

Appendix (continued)

\begin{tabular}{|c|c|c|c|}
\hline Name & Location & Role & Contribution \\
\hline $\begin{array}{l}\text { Nikoletta } \\
\text { Kollia, MD }\end{array}$ & $\begin{array}{l}\text { "Attikon" University } \\
\text { Hospital, Athens, } \\
\text { Greece }\end{array}$ & Author & $\begin{array}{l}\text { Data collection, } \\
\text { critical comments } \\
\text { during } \\
\text { manuscript } \\
\text { revision }\end{array}$ \\
\hline $\begin{array}{l}\text { Konstantinos } \\
\text { Voumvourakis, } \\
\text { MD, PhD }\end{array}$ & $\begin{array}{l}\text { "Attikon" University } \\
\text { Hospital, Athens, } \\
\text { Greece }\end{array}$ & Author & $\begin{array}{l}\text { Data collection, } \\
\text { critical comments } \\
\text { during } \\
\text { manuscript } \\
\text { revision }\end{array}$ \\
\hline $\begin{array}{l}\text { Georgios } \\
\text { Tsivgoulis, MD, } \\
\text { PhD, MSc, FESO }\end{array}$ & $\begin{array}{l}\text { "Attikon" University } \\
\text { Hospital, Athens, } \\
\text { Greece; The } \\
\text { University of } \\
\text { Tennessee Health } \\
\text { Science Center, } \\
\text { Memphis }\end{array}$ & Author & $\begin{array}{l}\text { Data collection, } \\
\text { drafting the } \\
\text { manuscript }\end{array}$ \\
\hline
\end{tabular}

\section{References}

1. Salvarani C, Morris JM, Giannini C, et al. Imaging findings of cerebral amyloid angiopathy, $A \beta$-related angiitis (ABRA), and cerebral amyloid angiopathy-related inflammation: a single-institution 25-year experience. Medicine 2016;95:e3613.

2. Chung KK, Anderson NE, Hutchinson D, Synek B, Barber PA. Cerebral amyloid angiopathy related inflammation: three case reports and a review. J Neurol Neurosurg Psychiatry 2011;82:20-26. 


\section{Neurology}

\section{Teaching NeuroImages: Acute convexity subarachnoid hemorrhage: An underrecognized presentation of CAA-ri}

Aikaterini Theodorou, Stefanos Lachanis, Palaiologos Alexopoulos, et al. Neurology 2019;93; $524-\mathrm{e} 525$

DOI 10.1212/WNL.0000000000007873

\section{This information is current as of July 29, 2019}

\section{Updated Information \& Services}

References

Subspecialty Collections

Permissions \& Licensing

Reprints including high resolution figures, can be found at: http://n.neurology.org/content/93/5/e524.full

This article cites 2 articles, 1 of which you can access for free at: http://n.neurology.org/content/93/5/e524.full\#ref-list-1

This article, along with others on similar topics, appears in the following collection(s):

Hallucinations

http://n.neurology.org/cgi/collection/hallucinations MRI

http://n.neurology.org/cgi/collection/mri

Subarachnoid hemorrhage

http://n.neurology.org/cgi/collection/subarachnoid_hemorrhage

Information about reproducing this article in parts (figures,tables) or in its entirety can be found online at:

http://www.neurology.org/about/about_the_journal\#permissions

Information about ordering reprints can be found online:

http://n.neurology.org/subscribers/advertise

Neurology ${ }^{\circledR}$ is the official journal of the American Academy of Neurology. Published continuously since 1951, it is now a weekly with 48 issues per year. Copyright (O 2019 American Academy of Neurology. All rights reserved. Print ISSN: 0028-3878. Online ISSN: 1526-632X.

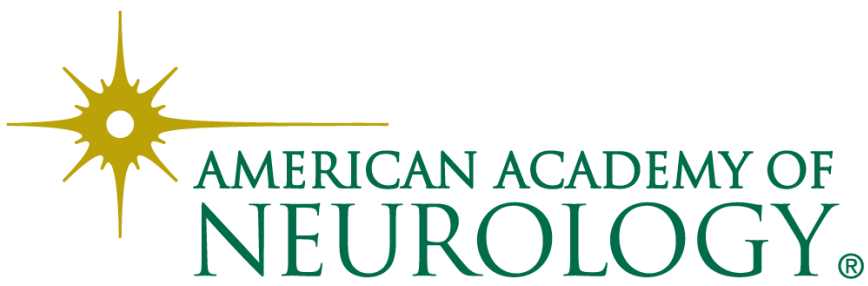

